\title{
HÍBRIDOS TRILINEALES DE MAÍZ COMUNES Y DE ALTA CALIDAD DE PROTEÍNA PARA VERACRUZ, MÉXICO ${ }^{1}$
}

\author{
Artemio Palafox-Caballero ${ }^{2}$, Oscar Hugo Tosquy-Valle ${ }^{2}$, Mauro Sierra-Macías ${ }^{2}$, Andrés Zambada-Martínez ${ }^{3}$, \\ Hugo Córdova-Orellana 4
}

\begin{abstract}
RESUMEN
Híbridos trilineales de maíz comunes y de alta calidad de proteína para Veracruz, México. Durante el 2004 se establecieron experimentos en los municipios de Medellín de Bravo, Camarón de Tejeda, Tlalixcoyan y San Andrés Tuxtla, en el estado de Veracruz. El objetivo de este trabajo fue conocer el comportamiento agronómico de híbridos trilineales comunes y con alta calidad de proteína (QPM), para identificar los más rendidores y de mejor adaptación. Así, fueron evaluados 11 híbridos QPM, siete comunes y los testigos H-519C de alta calidad y H-520 común, bajo diseño alpha látice $5 \times 4$ con tres repeticiones en parcelas de dos surcos de $5 \mathrm{~m}$ de largo separados a $80 \mathrm{~cm}$. Se midió el rendimiento, días a floración, altura de planta y de mazorca, aspecto de planta y de mazorca y sanidad de mazorca. Con más de $8 \mathrm{t} /$ ha los mejores híbridos en Medellín fueron HC 1 y HC 2. En Camarón de Tejeda destacaron $\mathrm{HC} 4$ y HC 2, por presentar rendimientos de entre 8 y 9 t/ha. HC 7 y HC 2 sobresalieron en Tlalixcoyan con más de 6 t/ha. En San Andrés HC 1 y HC 4 fueron los híbridos más rendidores. Por su alto rendimiento promedio de localidades (mayor a $7 \mathrm{t} / \mathrm{ha}$ ), adaptación y buena expresión fenotípica en planta y mazorca, los mejores híbridos fueron: HC 2, HC 4 y HC 1.
\end{abstract}

Palabras clave: Zea mays L., cruzas triples, calidad de proteína, trópico húmedo.

\begin{abstract}
Common and high quality protein in three way maize hybrids for Veracruz, México. During spring and summer seasons of 2004, four experiments of three way quality protein maize (QPM) hybrids, were carried out in Camarón de Tejeda, Medellín de Bravo, Tlalixcoyan and San Andrés Tuxtla, State of Veracruz. The objective of this research was to characterize the yield and agronomic features of these hybrids, and identify those with best agronomic behavior. Eleven QPM, eight common hybrids and two checks were evaluated, distributed under an alpha lattice design $(5 \times 4)$ with three replications in plots of two rows $5 \mathrm{~m}$ long and $80 \mathrm{~cm}$ wide. Individual analysis for yield, days to tassel, days to silking, plant height and ear length, plant and ear aspect, and combined analysis for yield were conducted. The best hybrids in Medellín de Bravo were: $\mathrm{HC} 1$ y HC 2. In Camarón de Tejeda, HC 4 y HC 2, presented the best grain yield between 8 to $9 \mathrm{t} / \mathrm{ha}$. HC $7 \mathrm{y}$ HC 2 were the best hybrids in Tlalixcoyan with more than 6 t/ha. In San Andres Tuxtla, HC 1 y HC 4 registered the highest grain yield. Across the four locations, the best hybrids considering grain yield, adaptation, and plant and ear agronomic characteristics were: HC 2, HC 4 y HC 1.
\end{abstract}

Key words: Zea mays L., three way hybrids, quality protein, humid tropic.

\section{INTRODUCCIÓN}

En México, el maíz ocupa el $40 \%$ de la superficie agrícola cultivable y es el alimento básico más importante que proporciona en promedio $39 \%$ de proteína asimilable y $59 \%$ de la energía que ingieren los mexicanos (Sierra et al. 2004). En el estado de Veracruz, la superficie anual establecida con este cereal supera las 530.000 ha (Palafox et al. 2005), de las cuales, 396.000 pertenecen a la región tropical. Con base a la

\footnotetext{
1 Recibido: 5 de agosto, 2005. Aceptado: 10 de mayo, 2006.

2 Campo Experimental Cotaxtla. INIFAP. Km. 34 Carret. Veracruz-Córdoba. Apdo. Postal 429, C.P. 91700. Veracruz, México. Tel: (01 229) 9342926, E. mail: palafox012@hotmail.com

3 Campo Experimental Papaloapan. INIFAP. Km. 66 Carret. Ciudad Alemán-Sayula. Apdo. Postal43, C.P. 95641 Isla Veracruz, México. Tel: (01 28187) 21682.

4 Centro Internacional de Mejoramiento de Maíz y Trigo (CIMMYT). Apdo. Postal 6-641, C.P. 06600, México D.F.
} 
profundidad del suelo, la disponibilidad de humedad para el cultivo, contenido de materia orgánica, pendiente del terreno, entre otros factores de diagnóstico, existen 200.000 ha que presentan los mejores niveles de estos factores y se clasifican con alto potencial para producir maíz (Tosquy et al. 1998). En esta superficie, aunque tradicionalmente se siembra maíz, se obtienen rendimientos muy bajos, de alrededor de $2 \mathrm{t} / \mathrm{ha}$ (Cano et al. 2001). Por su alto potencial productivo, en la superficie referida se recomienda el uso de híbridos, en los cuales se aprovecha el efecto heterótico de cruzar progenitores de relativa divergencia genética (Melchinger 1997).

Los híbridos formados por tres líneas representan una buena alternativa, ya que en ellos se tiene la ventaja de aprovechar como hembra una cruza simple de alto rendimiento (Rodríguez et al. 1997 y Sierra et al. 1998). Por otra parte, debido a la necesidad de mejorar la dieta básica de la población rural que depende en gran parte del consumo de este cereal, es deseable que se produzcan maíces más nutritivos, con mayor cantidad o calidad de proteína (Gómez et al. 2003). Los aminoácidos lisina y triptofano contenidos en el grano de maíz, son esenciales para el crecimiento y desarrollo humano (CIMMYT 2001); éstos se encuentran en mayor proporción en los maíces de alta calidad de proteína (QPM), derivados del aprovechamiento del gene mutante opaco 2, expresado en su versión homocigótica recesiva (Mertz et al. 1964). El contenido medio de proteína del maíz normal, del opaco- 2 y de los genotipos de alta calidad de proteína, es de 9,$1 ; 8,9$ y 9,8 por ciento, respectivamente, mientras que los valores de lisina son de 3,4; 4,4 y 4,08\%; asimismo, el contenido de triptofano es de 0,$59 ; 0,73$ y 0,75 por ciento (Mertz 1994). Por esta razón, el programa de maíz del Campo Experimental Cotaxtla del INIFAP en colaboración con el CIMMYT han generado híbridos de maíz comunes y con alta calidad de proteína, los cuales han mostrado buen comportamiento agronómico en el sureste de México (Hidalgo et al. 1998 y Tosquy et al. 2005). En seguimiento a estos trabajos de investigación, se estableció un experimento de híbridos trilineales élite repetido en cuatro localidades del estado de Veracruz, con el objetivo de conocer su comportamiento en regiones tropicales representativas e identificar los genotipos más rendidores y de mejor adaptación.

\section{MATERIALES Y MÉTODOS}

El experimento se estableció durante el ciclo primavera-verano 2004, en condiciones de temporal, en las localidades de Mata de Agua, municipio de Camarón de Tejeda, Campo Experimental Cotaxtla, municipio de Medellín de Bravo y La Torrecilla, municipio de Tlalixcoyan, en la parte central del estado de Veracruz y Axochio, municipio de San Andrés Tuxtla, en el sur de la entidad. El tipo de clima que prevalece en la primera localidad es $\mathrm{Aw}_{0}$ (el más seco de los subhúmedos) y en la segunda y tercera es $\mathrm{Aw}_{1}$ (intermedio en humedad entre $A w_{0}$ y $A w_{2}$ ), que corresponden a climas cálidos subhúmedos con lluvias en verano. En la localidad del sur el clima es Am, cálido húmedo con lluvias en verano y con 5 a $10 \%$ de lluvia invernal, de acuerdo a la clasificación de Köppen, modificada por García (1981). En las localidades Mata de Agua y Campo Experimental Cotaxtla los experimentos se establecieron en suelos de textura migajón arcilloso, con pH de 6,5; en La Torrecilla y Axochío, en suelos de textura franca con pH de 6,2 (López 1998).

Se evaluaron 11 híbridos de alta calidad de proteína (QPM) y siete híbridos comunes (Cuadro 1). Las líneas progenitoras de estos híbridos trilineales provienen del programa tropical de maíz del Centro

Cuadro 1. Relación de híbridos de maíz evaluados y su descripción de acuerdo a su calidad de proteína. México, 2004.

\begin{tabular}{lcc}
\hline \multicolumn{1}{c}{ Genotipo } & $\begin{array}{c}\text { Nivel de } \\
\text { proteína }\end{array}$ & $\begin{array}{c}\text { Código } \\
\text { Identi- } \\
\text { ficación }\end{array}$ \\
\hline (CML448 x CML476) x CML449 & Común & HC 1 \\
(CML449 x CML448) x CL-PWSD05 & Común & HC 2 \\
(CL-02181 x CML269) x CL-RCW42 & Común & HC 3 \\
(CML449xCML448) x CL-PWSD03 & Común & HC 4 \\
(CML448 x CML449) x CL-RCW36 & Común & HC 5 \\
(CML449 x CML448) x CML450 & Común & HC 6 \\
(CML398 x CML269) x CL-RCW42 & Común & HC 7 \\
(CML492 x CML146) x CLQ-RCWQ50 & QPM & HQ 1 \\
(CML264Q x CML492) x CML491 & QPM & HQ 2 \\
(CML264Q x CML159) x CML491 & QPM & HQ 3 \\
(CML492 x CML150) x CML491 & QPM & HQ 4 \\
(CML264Q x CML150) x CML491 & QPM & HQ 5 \\
(CML247 x CML2) x R1 & QPM & HQ 6 \\
(CML264Q x CML491) x CML492 & QPM & HQ 7 \\
(CML492 x CML150) x CML264Q & QPM & HQ 8 \\
(CML144 x CML159) x CML 491 & QPM & HQ 9 \\
(CML144 x CML147) x CML264Q & QPM & HQ 10 \\
(CML144 x CML147) x CML491 & QPM & HQ 11 \\
Testigo H-520 & Común & H-520 \\
Testigo H-519C & QPM & H-519C \\
\hline
\end{tabular}

HC = Híbrido Común; HQ = Híbrido de alta calidad de proteína (QPM).

AGRONOMÍA MESOAMERICANA 17(2): 201-206. 2006 
Internacional de Mejoramiento de Maíz y Trigo (CIMMYT). Se utilizaron como testigos los híbridos comerciales H-519C, de alta calidad de proteína y H520 con niveles comunes de lisina y triptofano, formados por el programa de mejoramiento genético de maíz del INIFAP en Veracruz, México.

El diseño estadístico utilizado fue Alpha látice $5 \mathrm{x}$ 4 con tres repeticiones. Los ensayos regionales se establecieron en surcos separados a $80 \mathrm{~cm}$, con una planta cada $20 \mathrm{~cm}$, lo que da una densidad de 62.500 plantas por hectárea; éstos se condujeron de acuerdo a las prácticas tecnológicas recomendadas por el INIFAP para cada región (Tinoco et al. 2002).

Como variables de respuesta se consideraron: rendimiento de grano (RG en t/ha), ajustado al $12 \%$ de humedad; días a floración media (FM en d), días contados a partir de la siembra al momento en que el $50 \%$ de las plantas se encontraban emitiendo polen, altura de planta (ALT P) y de mazorca (ALT M), medidas en cinco plantas al azar de cada parcela útil, desde la superficie del suelo hasta la base de la espiga y del nudo donde se inserta la mazorca principal, respectivamente; aspecto de planta (ASP P) y aspecto de mazorca (ASP M), ambas evaluadas mediante una escala de uno a cinco, donde uno representó a las plantas de buen vigor, uniformes en altura y posición de mazorca y con mazorcas uniformes en tipo, tamaño y con buena cobertura y cinco correspondió a las plantas con características inadecuadas, como muy variables en altura y posición de mazorca, con escaso vigor y con mazorcas irregulares en tamaño y forma, y de mala cobertura; así como sanidad de mazorca (SAN M), evaluada también mediante una escala de uno a cinco, donde uno representó a mazorcas completamente sanas y cinco mazorcas totalmente podridas (CIMMYT 1987). Mediante el paquete estadístico de la Universidad Autónoma de Nuevo León (Olivares 1994) se realizaron análisis individuales para todas las variables y análisis combinado para rendimiento de grano y donde se detectó significancia, para la separación de promedios se aplicó la prueba de la Diferencia Mínima Significativa (DMS). También se hicieron correlaciones simples para conocer el grado de asociación entre las diferentes variables agronómicas y el rendimiento de grano (Olivares 1994).

\section{RESULTADOS Y DISCUSIÓN}

Los análisis individuales del rendimiento de grano detectaron diferencias significativas en todas las

ISSN: 1021-7444 localidades de prueba. En el Cuadro 2 se visualiza que de acuerdo a la DMS, en la localidad de Cotaxtla sobresalieron siete genotipos con rendimientos superiores a 7,4 t/ha en los que se incluye al testigo H-520. En este grupo destacan los híbridos comunes HC 1 y HC 2 , los cuales presentaron los mayores rendimientos. En Mata de Agua, aunque cuatro híbridos obtuvieron un rendimiento significativamente superior al resto de los tratamientos, los híbridos comunes HC 4 y HC 2 sobresalieron por su mayor capacidad productiva, superando en promedio en $42,1 \%$ el rendimiento obtenido por los testigos regionales. En la localidad de La Torrecilla el grupo superior estuvo formado por ocho híbridos, incluido el testigo H-520 común; de estos, sólo HC 7 y HC 2 lograron rendimientos superiores a 6 t/ha. A su vez, en Axochío nueve híbridos experimentales resultaron estadísticamente superiores, pero sólo HC 1 y HC 4 superaron las 6 t/ha. Estos resultados concuerdan con lo encontrado por Tosquy et al. (2005) que al evaluar bajo condiciones de temporal un grupo de genotipos experimentales de maíz en San Andrés Tuxtla, detectaron 10 híbridos QPM sobresalientes,

Cuadro 2. Rendimiento de grano ( $\mathrm{t} / \mathrm{ha}$ ) de híbridos trilineales, promedio de localidades. México, 2004.

\begin{tabular}{lccccc}
\hline Genotipo & Cotaxtla & $\begin{array}{c}\text { Mata de } \\
\text { Agua }\end{array}$ & $\begin{array}{c}\text { La } \\
\text { Torrecilla }\end{array}$ & Axochio Promedio \\
\hline HC 2 & 8.20 & 8,73 & 6,24 & 5,59 & $7,19^{*}$ \\
HC 4 & 7,69 & 9,06 & 5,96 & 6,03 & $7,19^{*}$ \\
HC 1 & 8,26 & 7,62 & 5,84 & 6,31 & $7,08^{*}$ \\
HC 3 & 7,70 & 7,96 & 4,67 & 5,81 & 6,54 \\
HC 6 & 7,42 & 8,12 & 5,04 & 4,91 & 6,37 \\
HC 7 & 7,22 & 6,80 & 6,31 & 4,99 & 6,33 \\
HQ 1 & 6,83 & 7,36 & 4,94 & 5,93 & 6,27 \\
HC 5 & 7,49 & 6,18 & 5,40 & 5,63 & 6,18 \\
H-520 & 7,66 & 6,91 & 5,36 & 4,24 & 6,04 \\
HQ 5 & 6,51 & 6,32 & 5,14 & 5,50 & 5,87 \\
HQ 3 & 6,80 & 6,67 & 5,07 & 4,60 & 5,79 \\
HQ 4 & 6,54 & 6,68 & 4,18 & 5,71 & 5,78 \\
HQ 2 & 6,82 & 6,60 & 3,88 & 5,06 & 5,59 \\
HQ 8 & 5,93 & 7,36 & 4,57 & 4,48 & 5,59 \\
HQ 6 & 6,38 & 5,18 & 4,77 & 5,71 & 5,51 \\
HQ 7 & 6,14 & 6,64 & 2,79 & 4,72 & 5,07 \\
HQ 10 & 5,07 & 6,06 & 4,08 & 4,70 & 4,98 \\
H-519C & 5,78 & 5,61 & 2,28 & 3,74 & 4,35 \\
HQ 9 & 5,19 & 4,95 & 3,51 & 2,25 & 3,98 \\
HQ 11 & 4,31 & 3,50 & 3,01 & 3,18 & 3,50 \\
\hline Promedio & $6,70 *$ & $6,72 *$ & 4,65 & 4,97 & 5,76 \\
DMS & 0,092 & 1,28 & 1,24 & 0,98 & \\
\hline
\end{tabular}

* = Significancia al 0,05 de probabilidad de error para genotipos y localidades.

AGRONOMÍA MESOAMERICANA 17(2): 201-206. 2006 
con rendimientos superiores a $6,2 \mathrm{t} / \mathrm{ha}$, los cuales superaron significativamente a estos mismos testigos.

El análisis de varianza combinado para rendimiento de grano detectó diferencias significativas entre localidades, genotipos y la interacción de ambos factores. El coeficiente de variación fue de 11,62\%, que indica que los resultados son confiables y que hubo alta eficiencia en el manejo de los experimentos. La significancia detectada entre localidades, señala que los ambientes de evaluación presentan diferencias en sus condiciones agroecológicas y en su potencial para producir maíz, lo cual se observa en el Cuadro 2, donde los mayores rendimientos por localidad se obtuvieron en Mata de Agua y Cotaxtla que presentaron un rendimiento promedio mayor a $6,7 \mathrm{t} / \mathrm{ha}$, significativamente superior al de las otras dos localidades.

En el factor genotipos, los híbridos experimentales comunes HC 2, HC 4 y HC 1 resultaron sobresalientes estadísticamente con rendimientos mayores a 7,0 t/ha. Estos genotipos en promedio superaron en 18,3 y 64,3\% el rendimiento de los testigos H-520 común y H-519 C de alta calidad de proteína, respectivamente. El efecto significativo detectado en la interacción localidad por genotipo indicó que el comportamiento productivo de alguno de estos híbridos varió de una localidad a otra. Así por ejemplo, mientras HC 1 obtuvo rendimientos significativamente mayores en las localidades de Cotaxtla, La Torrecilla y Axochio, no fue sobresaliente en la localidad de Mata de Agua. Por su parte, los híbridos HC 7 y HQ 1, que fueron sobresalientes en las localidades de La Torrecilla y Axochio, respectivamente, en las otras localidades presentaron rendimientos inferiores con respecto al grupo sobresaliente. El testigo común H-520 se ubicó en el grupo superior en las localidades de Cotaxtla y La Torrecilla, en tanto que el testigo H-519C, que interaccionó menos con el ambiente, obtuvo bajos rendimientos en las cuatro localidades (Cuadro 2).

$\mathrm{Al}$ establecer experimentos en diferentes ambientes, se espera que el comportamiento de los genotipos en evaluación sea diferente en alguno de ellos en particular, o en general, en todos los ambientes (Cruz 1989). Los resultados de estos trabajos permitieron identificar híbridos experimentales de maíz que presentaron buena respuesta en diferentes ambientes y que además tienen mayor potencial de rendimiento que los testigos regionales; por lo que representan nuevas y mejores opciones para los productores.

El Cuadro 3 muestra las características agronómicas de los híbridos evaluados en las localidades de prueba, donde se observa que en general todos los genotipos se comportaron como de ciclo vegetativo intermedio, con una diferencia en su floración de 4,4 días entre el mayor y el menor valor. En este carácter lo deseable es disponer de genotipos cuyas floraciones durante el ciclo primavera-verano se presenten antes de los 60 días (Tosquy et al. 2005). Referente a las variables altura de planta y mazorca, los híbridos presentaron valores inferiores a 2,5 y 1,5 m de altura de planta y de mazorca, respectivamente; que son deseables, ya que la mayoría de los materiales comerciales que se siembran comúnmente en la región son más altos, lo cual es desfavorable debido a que se incrementan los riesgos de acame del cultivo por efecto de los vientos (Sierra 2002). Estas variables mostraron una correlación positiva y significativa con el rendimiento de grano, que indican en general que a mayor altura se tuvieron mayores rendimientos. En cuanto al aspecto de planta, la mejor expresión correspondió al testigo H-519C y al HC 3, aunque en general todos los híbridos manifestaron buen aspecto, dado que los híbridos experimentales evaluados ya han sido seleccionados en ensayos preliminares. Las variables aspecto y sanidad de mazorca fueron las que más se asociaron negativamente con el rendimiento de grano $\left(\mathrm{r}=-0,86^{* *}\right.$ y $\left.\mathrm{r}=-0,75^{* *}\right)$. En este sentido los híbridos que obtuvieron los mayores rendimientos mostraron las mejores calificaciones de estas dos variables; en contraste con algunos híbridos experimentales y el testigo $\mathrm{H}-519 \mathrm{C}$ que presentaron valores altos, que son menos favorables en aspecto y sanidad de mazorca y bajos rendimientos de grano.

\section{CONCLUSIONES}

Por su alto rendimiento, los mejores híbridos para Cotaxtla, municipio de Medellín de Bravo, fueron: HC 1 y HC 2. Para Mata de Agua, municipio de Camarón de Tejeda, los híbridos comunes HC 4 y HC 2 fueron los más rendidores. En La Torrecilla, Tlalixcoyan, HC 7 y $\mathrm{HC} 2$ resultaron las mejores alternativas tecnológicas. En Axochio, municipio de San Andrés Tuxtla, los híbridos más productivos fueron $\mathrm{HC} 1$ y HC 4 . Por su alto 
Cuadro 3. Características agronómicas y rendimiento promedio de híbridos trilineales de maíz, promedio de cuatro localidades. México, 2004.

\begin{tabular}{lccccccc}
\hline Genotipo & $\begin{array}{c}\text { FM } \\
\text { (d) }\end{array}$ & $\begin{array}{c}\text { ALT } \\
\mathbf{P}\end{array}$ & $\begin{array}{c}\text { ALT } \\
\mathbf{M}\end{array}$ & $\begin{array}{c}\text { ASP } \\
\mathbf{P}\end{array}$ & $\begin{array}{c}\text { ASP } \\
\mathbf{M}\end{array}$ & $\begin{array}{c}\text { SAN } \\
\mathbf{M}\end{array}$ & $\begin{array}{c}\text { RG } \\
(\mathbf{t} / \mathbf{h a})\end{array}$ \\
\hline HC 2 & 56,2 & 230,52 & 128,2 & 2,56 & 1,72 & 1,90 & 7,19 \\
HC 4 & 56,9 & 221,6 & 111,7 & 2,37 & 1,81 & 1,72 & 7,19 \\
HC 1 & 54,4 & 214,8 & 110,4 & 2,55 & 1,60 & 1,63 & 7,08 \\
HC 3 & 57,0 & 236,9 & 117,7 & 2,16 & 2,13 & 2,11 & 6,54 \\
HC 6 & 54,5 & 211,4 & 106,0 & 2,54 & 2,28 & 1,95 & 6,37 \\
HC 7 & 58,8 & 220,0 & 105,5 & 2,46 & 2,22 & 2,28 & 6,33 \\
HQ 1 & 55,7 & 236,8 & 107,2 & 2,20 & 2,39 & 2,40 & 6,27 \\
HC 5 & 56,0 & 227,3 & 117,2 & 2,28 & 2,21 & 2,03 & 6,18 \\
H-520 & 54,6 & 205,9 & 104,9 & 2,42 & 2,03 & 1,85 & 6,04 \\
HQ 5 & 55,9 & 205,8 & 101,1 & 2,27 & 2,20 & 2,22 & 5,87 \\
HQ 3 & 56,0 & 208,8 & 108,8 & 2,23 & 2,27 & 2,17 & 5,79 \\
HQ 4 & 56,0 & 210,4 & 107,3 & 2,42 & 2,14 & 2,01 & 5,78 \\
HQ 2 & 56,3 & 206,7 & 98,2 & 2,40 & 2,14 & 2,14 & 5,59 \\
HQ 8 & 55,2 & 196,4 & 85,4 & 2,68 & 2,88 & 2,96 & 5,59 \\
HQ 6 & 55,1 & 218,4 & 108,3 & 2,38 & 2,18 & 2,17 & 5,51 \\
HQ 7 & 55,2 & 200,9 & 89,9 & 2,59 & 2,49 & 2,32 & 5,07 \\
HQ 10 & 56,4 & 216,8 & 95,2 & 2,44 & 2,73 & 2,84 & 4,98 \\
H-519C & 55,6 & 225,2 & 118,4 & 2,10 & 2,57 & 2,60 & 4,35 \\
HQ 9 & 56,6 & 193,9 & 101,0 & 2,71 & 2,93 & 2,51 & 3,98 \\
HQ 11 & 57,0 & 196,6 & 100,5 & 2,73 & 3,00 & 2,78 & 3,50 \\
\hline Promedio & 55,97 & 215,22 & 106,23 & 2,42 & 2,296 & 2,229 & 5,76 \\
\hline Coef. Det. & & & & & & & \\
RG vs X & $\mathrm{r}=-0,09 \mathrm{~ns}$ & $\mathrm{r}=0,56 * *$ & $\mathrm{r}=0,45$ & $\mathrm{r}=-0,27 \mathrm{~ns}$ & $\mathrm{r}=-0,86 * *$ & $\mathrm{r}=-0,75 * *$ & \\
\hline
\end{tabular}

$\mathrm{RG}=$ Rendimiento de grano; $\mathrm{X}=$ Cada una de las características agronómicas. $\mathrm{ns}=$ No significativo. $* *=$ Significancia de la correlación al 0,01 de probabilidad de error.

rendimiento, adaptación y buena expresión fenotípica en planta y sanidad de mazorca, los mejores híbridos a través de localidades fueron: HC 2, HC 4 y HC 1.

\section{LITERATURA CITADA}

CANO, O.; TOSQUY, O.; SIERRA, M.; RODRÍGUEZ, F. 2001. Fertilización y densidad de población en genotipos de maíz cultivados bajo condiciones de temporal. Agronomía Mesoamericana 12(2): 199-203.

CENTRO INTERNACIONAL DE MEJORAMIENTO DE MAÍZ Y TRIGO (CIMMYT). 1987. Manejo de ensayos e informe de datos para el programa de ensayos internacionales de maíz del CIMMYT. México, D. F. 23 p.

CENTRO INTERNACIONAL DE MEJORAMIENTO de MAÍZ Y TRIGO (CIMMYT). 2001. The quality protein maize revolution. Improved nutrition and livelihoods for the poor. México, D. F. 7 p.
CRUZ, R. 1989. Un ejemplo de la prueba exacta de los parámetros de estabilidad de Eberhart y Russell. Fitotecnia Mexicana 12(2): 147-155.

GARCÍA, E. 1981. Modificaciones al sistema de clasificación climática de Köppen. Instituto de Geografía de la UNAM. $3^{\text {a }}$ ed. México. 252 p.

GÓMEZ, N.; SIERRA M.; CANTÚ, M.; RODRÍGUEZ, F.; MANJARRES, M.; GONZÁLEZ, M.; ESPINOSA, A.; BETANZOS, E.; CÓRDOVA, H.; CABALLERO, F.; TURRENT, A.; GARCÍA, A.; RAMÍREZ, G.; SANDOVAL, A.; COUTIÑO, B.; CERVANTES, E.; REYES, C.; NAVA, L. 2003. V-537C y V-538C, nuevas variedades de maíz con alta calidad de proteína para el trópico mexicano. Fitotecnia Mexicana 26 (3): 213-214.

HIDALGO, H.; JEFFERS, D.; CASTAÑÓN, G.; RODRÍGUEZ, F. 1998. Resistencia al achaparramiento del maíz mediante infestaciones de Dalbulus maydis en maíz. Agronomía Mesoamericana 9(2): 119-124. 
LÓPEZ, J. 1998. Interpretación de los resultados de los análisis químicos de suelos agrícolas. Ed. Colegio de Postgraduados. Instituto de Fitosanidad. Manlio Fabio Altamirano, Veracruz, México. 45 p.

MELCHINGER, E. 1997. Genetic diversity and heterosis. In: J. G. Coors; S. Pandey. eds. Memoria the internacional symposium about the genetics and exploitation of heterosis in crops. Mexico city, CIMMYT. México. p. $99-118$.

MERTZ, T.; BATES, S.; NELSON, F. 1964. Mutant gene that changes protein composition and increase lysine content of maize endosperm. Science 145: 279.

MERTZ, T. 1994. Thirty years of Opaque-2 maize. In: B. A. Larkins and E. T. Mertz. eds. Proceedings of the of international symposium on quality protein maize. Ed. EMBRAPA/CNPMS, Sete Lagoas, Brazil. p 1-9.

OLIVARES, E. 1994. Paquete estadístico de diseños experimentales [Programa de cómputo]. Facultad de Agronomía de la Universidad Autónoma de Nuevo León. Marín, Nuevo León, México. Disco compacto.

PALAFOX, A.; TOSQUY, O. H.; SIERRA, M.; TURRENT, A.; ESPINOSA, A. 2005. Respuesta de híbridos de maíz normales y con alta calidad de proteína a la fertilización química. TERRA Latinoamericana 23(1): 129-135.

RODRÍGUEZ, F.; SIERRA, M.; CANO, O.; CASTAÑÓN, G. 1997. Tree way crosses as an alternative for producing Maite in Veracruz, México. In: J. G. Coors and S. Pandey. eds. Memoria The International Symposium about the genetics and exploitation of heterosis in crops. Mexico City, Mexico. p. 238
SIERRA, M.; RODRÍGUEZ, F.; CANO, O. 1998. Híbridos trilineales de maíz A. usando como probadores las cruzas simples H-513, CML247 x CML254 y LRB14 x D-539. In: H. Barradas ed. Memoria de la XI Reunión Científica Tecnológica, Forestal y Agropecuaria de Veracruz. Veracruz, Ver., México. p. 133-139.

SIERRA, M. 2002. Uso de probadores en la selección de líneas para formar híbridos de maíz (Zea mayz L.). Tesis de Doctorado en Ciencias. Tecomán, Colima, México. $177 \mathrm{p}$.

SIERRA, M.; PALAFOX, A.; CANO, O.; RODRÍGUEZ, F.; ESPINOSA, A.; TURRENT, A.; GÓMEZ, N.; CÓRDOVA, H.; VERGARA, N.; AVELDAÑO, R.; SANDOVAL, J.; BARRÓN, S.; ROMERO, J.; CABALLERO, F.; GONZÁLEZ, M.; BETANZOS, E. 2004. H-553C, híbrido de maíz de calidad proteínica para el trópico húmedo de México. Fitotecnia Mexicana 27 (1): 117-119.

TINOCO, A.; RODRÍGUEZ, A.; SANDOVAL, A.; BARRÓN, S.; PALAFOX, A., ESQUEDA V.; SIERRA, M.; ROMERO, J. 2002. Manual de producción de maíz para los estados de Veracruz y Tabasco. Libro Técnico Núm. 9. INIFAP. CIRGOC. Campo Experimental Papaloapan. Veracruz, México. 113 p.

TOSQUY, O.; DE LA GARZA, R.; CASTAÑÓN, G.; MORONES, R. 1998. Fertilización edáfica y densidades de población para producción de semilla de líneas de maíz. Agricultura Técnica en México 24(2): 111-119.

TOSQUY, O.; PALAFOX, A.; SIERRA, M.; ZAMBADA, A.; MARTÍNEZ, R.; GRANADOS, G. 2005. Comportamiento agronómico de híbridos de maíz en dos municipios de Veracruz, México. Agronomía Mesoamericana 16(1): 7-12. 